\title{
BIFURCATION AND STABILITY
}

\section{IN YANG-MILLS THEORY WITH SOURCES *}

\section{R. Jackiw}

Center for Theoretical Physics.
Laboratory for Nuclear Science and Department of Physics Massachusetts Institute of Technology
Cambridge, Massachusetts 02139

*

This work is supported in part through funds provided by the U.S. DEPARTMENT OF ENERGY (DOE) under contract EY-76-C-02-3069.

\author{
Summer Workshop on Theoretical Physics \\ ICTP \\ Trieste, Italy \\ 12 July-3 August, 1979 \\ Durham Symposium on Geometry and Physics \\ Durham University \\ Durham, England \\ 11-2l July, 1979
}




\section{DISCLAIMER}

This report was prepared as an account of work sponsored by an agency of the United States Government. Neither the United States Government nor any agency Thereof, nor any of their employees, makes any warranty, express or implied, or assumes any legal liability or responsibility for the accuracy, completeness, or usefulness of any information, apparatus, product, or process disclosed, or represents that its use would not infringe privately owned rights. Reference herein to any specific commercial product, process, or service by trade name, trademark, manufacturer, or otherwise does not necessarily constitute or imply its endorsement, recommendation, or favoring by the United States Government or any agency thereof. The views and opinions of authors expressed herein do not necessarily state or reflect those of the United States Government or any agency thereof. 


\section{DISCLAIMER}

Portions of this document may be illegible in electronic image products. Images are produced from the best available original document. 
CONTENTS

I. INTRODUCTION

II. SOLUTIONS TO YANG-MILLS THEORY WITH SOURCES

A. Preliminaries

B. Arbitrary Sources

C. Sources with Critical strength

III. STABILITY IN DYNAMICAL SYSTEMS

A. Review of the General Theory

B. Stability Analysis for Yang-Mills Theory

1. Abelian Coulomb Solution

2. Non-Abelian Coulomb Solution

3. Bifurcating Solutions

IV. CONCLUSION

REFERENCES

FIGURE CAPTIONS 


\section{INTRODUCTION}

In my lecture I shall discuss some recent work on solutions to classical Yang-Mills theory. The investigations that I shall summarize study the field equations with static, external [nondynamical] sources./ The physical, quantum-mechanical significance of such solutions has not thus far been as profound as that of solitons, where sources are dynamical i.e. monopoles with Higgs-field sources; nor as that of instantons, where sources are absent but the equations are continued to imaginary time. Nonetheless the new results are interesting in their differences from the Abelian counterpart and should suggest intuition about the physical content of non-Abelian gauge-quantum field theory. Moreover, structurally the equations are sufficiently intricate to provide a most interesting example of analysis in mathematical physics.

There is now available a variety of solutions for review; but only recently did a pattern emerge which allows for a comprehensive description. A summary of this is presented in Section II. Section III is devoted to an account of stability properties. I begin by recalling the theory of stability - a subject widely studied by physicists in former times, but now, in its general form, largely forgotten. The general theory does not rely on minima of the energy and is found to be applicable to the Yang-Mills model. It comes as no surprise that the non-Abelian structure lets the gauge theory share with a top the phenomenon of stable configurations which do not mini- 
$-2-$

mize the energy. The Section concludes with a description of work in progress which assesses the stability of various soltrons. Finally $\sqrt{a}$ list of open questions and problems for furthe research comprise the concluding section IV.

II. SOLUTIONS TO YANG-MILLS THEORY WITH SOURCES

A. Preliminaries

The field equations with which we are concerned are

$$
\begin{aligned}
& D_{\mu} F^{\mu \nu}=\delta^{\nu \rho} \rho \\
& F^{\mu \nu}=\partial^{\mu} A^{\nu}-\partial^{\nu} A^{\mu}+\left[A^{\mu}, A^{\nu}\right] \\
& D_{\mu}=\partial_{\mu}+\left[A_{\mu},\right.
\end{aligned}
$$

We study the SU(2) theory with coupling strength scaled to unity and use interchangeably component notation and antiHermitian matrix notation; e.g. $\rho_{a}, a=1,2,3 ; \rho=\rho_{a} \sigma^{a} / 2 i, \sigma^{a}=$ Pauli matrices. The source $\rho$ is taken to be a given, time-independent function, $\partial_{t} \rho=0$. Eq. (2.la) carries with it an integrability condition: the right-hand side must be covariantly conserved. In the present circumstance that requirement reduce to

$$
\left[A^{0}, \rho\right]=0
$$

The energy of the system is given by a positive, gaugeinvariant formula.

$$
\begin{aligned}
& \mathcal{E}=\frac{1}{2} \int d \vec{r}\left\{\vec{E}_{a}^{2}+\vec{B}_{a}^{2}\right\} \\
& E_{a}^{i}=F_{a}^{i 0} ; B_{a}^{i}=-\frac{1}{2} \varepsilon^{i j k} F_{a}^{j k}
\end{aligned}
$$


$-3-$

The class of solutions which I shall here be describing is delimited by the requirement of finite energy. This means that sources must also be well-behaved; a condition which will. not be spelled out in detail, beyond noting that point sources are excluded; $\rho$ is an extended function.

We shall need a Hamiltonian formulation for the field equations (2.1); since they are locally gauge invariant this is not straightforward. In order to overcome the familiar difficulty, we do not pick a gauge; rather we take the varations used for obtaining the equations of motion to be constrained by Gauss' law, which is the $\nu=0$ component of (2.1a). Specifically we take the Hamiltonian to coincide with $\mathcal{E}$, viewed as a functional of independent variables of $\vec{E}$ and $\vec{A}$, while $\vec{B}$ is constructed in the usual way from $\vec{A}$.

$$
\vec{B}_{a}=\vec{\nabla} \times \vec{A}_{a}-\frac{i}{2} \varepsilon_{a b c} \vec{A}_{b} \times \vec{A}_{c}
$$

$-\vec{E}$ is identified with the canonical momentum conjugate to $\vec{A}$, and the constraint of Gauss' law is imposed with the help of a Lagrange multiplier, here called $A_{a}^{\circ}$. Hence unrestricted varation can be performed on

$$
\bar{\varepsilon}=\varepsilon-\int d \vec{r} A_{a}^{0}\left(\vec{\nabla} \cdot \vec{E}_{a}-\varepsilon_{a b c} \vec{A}_{b} \cdot \vec{E}_{c}-\rho_{a}\right)_{(2.5)}
$$

In this way the Yang-Mills equations are obtained.

$$
O=-\frac{\delta \vec{Q}}{\delta A_{a}^{0}}=\overrightarrow{\nabla_{a}} \cdot \vec{E}_{a}-\varepsilon_{a b c} \vec{A}_{b} \cdot \vec{E}-\rho_{a}
$$

Gauss' law constraint 
$-4-$

$\partial_{t} \vec{E}_{a}=\frac{\delta \bar{\varepsilon}}{\delta \vec{A}_{a}}=\vec{\nabla} \times \vec{B}_{a}-\varepsilon_{a b c} \vec{A}_{b} \times \vec{B}_{c}-\varepsilon_{a b c} A_{b}^{0} \vec{E}_{c}$

Ampère's law

$\partial_{t} \vec{A}_{a}=-\frac{\delta \vec{\varepsilon}}{\delta \vec{E}_{a}}=-\vec{E}_{a}-\vec{\nabla} A_{a}^{0}+\varepsilon_{a b c} \vec{A}_{b} A_{c}^{0}$

Definition of $\overrightarrow{\mathrm{E}}_{\mathrm{a}}$

Note that static solutions [all time-derivatives vanish] are critical points of the energy, subject to the constraint of Gauss' law. 1,2

Presentation of solutions is complicated by the gauge covariance of $(2.1)$ : if $\mathrm{A}^{\mu}$ solves the equations with source $\rho$, then the equations with a gauge-rotated source $\rho^{\prime}$

$$
\rho^{\prime}=U^{-1} \rho U
$$

are solved by gauge transforming the previous.

$$
A^{\prime \mu}=U^{-1} A^{\mu} U^{\prime}+U^{-1} \partial^{\mu} U^{\prime}
$$

[Here $U$ is an SU(2) matrix.] Two solutions related as above describe the same physical situation and we shall view them as the same solution but presented in different "gauge frames". Frequently we shall speak of an "Abelian gauge frame" - one in which the source points in the third direction.

$$
p_{a}=\delta_{a 3} q
$$


of course results for gauge invariant quantities, like the energy, are frame independent.

In addition to the above gauge covariance, there is present also a gauge invariance with respect to gauge transformations which leave the source unchanged. From (2.2), we see that gauge transformations with $U$ constructed from $A^{\circ}$ are of this type. Thus it is always possible to pass to the temporal gauge where $A^{\circ}$ vanishes, without changing the gauge frame. Solutions naturally fall into two classes: those that exist for arbitrary sources and those that require a critical, finite source strength. We list these in turn.

\section{B. Arbitrary Sources}

Four different types of solutions will be discussed in this Section, two static, two time-dependent. The latter provide a well-defined generalization of the former.

The most obvious Yang-Mills solution is the static coulomb one which is readily presented in the Abelian gauge frame, where it is given by the regular solution to Poisson's equation. ${ }^{3}$

$$
\begin{aligned}
& A_{a}^{c}=\delta_{a 3} \varphi \\
& \vec{A}_{a}=0 \\
& q=-\frac{1}{\nabla^{2}} q
\end{aligned}
$$

An alternate description, still in the Abelian gauge frame, is gotten by passing to the temporal gauge. 


$$
\begin{aligned}
& A_{a}^{0}=0 \\
& \vec{A}_{a}=\delta_{a 3} \vec{\nabla} \varphi t
\end{aligned}
$$

The energy of this, according to (2.3), is the familiar coulomb expression.

$$
\Theta_{C}=\frac{1}{2} \int q \frac{-1}{\nabla^{2}} q=\frac{1}{8 \pi} \int d \vec{r}^{\prime} d \vec{r}^{\prime} \frac{q(\vec{r}) q\left(\vec{r}^{\prime}\right)}{\left|\vec{r}-\vec{r}^{\prime}\right|}
$$

Note that in the Abelian frame, the solution vanishes with the source.

The next solution is a time-dependent generalization of the above. It shares with the Coulomb solution the [gauge-invariant] property that the magnetic field vanishes. From Ampère's law it follows that, in the temporal gauge, vanishing $\vec{B}$ implies a static electric field. Eqs. (2.4) and (2.6c) require the electric field to be [gauge equivalent to] a gradient of a scalar [matrix] function $\Phi$, which further must satisfy

$$
[\vec{\nabla} \Phi, \vec{\nabla} \Phi]
$$

Thus we have, for $\Phi^{\prime}$ 's satisfying (2.12),

$$
\begin{aligned}
& A^{\prime}{ }^{\prime}=Q \\
& \vec{A}^{\prime}=\vec{\nabla} \Phi t
\end{aligned}
$$

The source which gives rise to such a field is determined by Gauss' law. 


$$
\nabla^{2} \Phi=-\rho^{\prime}
$$

There are as many configurations in the above category as there are functions $\Phi$ consistent with (2.12). However, our interest is only in those for which $\rho^{\prime}$ is gauge equivalent to the Abelian frame formula $(2.8)$; only then are we dealing with solutions to the same problem as the coulomb one.

$$
P^{\prime}=U \rho U^{-1}=U \frac{\sigma^{3}}{2 i} U^{-1} q
$$

When (2.14) holds, we can express the solution in the Abelian frame, in the temporal gauge.

$$
\begin{aligned}
& A^{0}=0 \\
& \vec{A}=-\vec{E} t-U^{-1} \vec{\nabla} U \\
& \vec{E}=-U^{-1} \vec{\nabla} \Phi U^{\prime}
\end{aligned}
$$

The energy of the above is given by a Coulomb-type formula.

$$
\mathcal{C}=\frac{1}{2} \int \rho_{a}^{\prime} \frac{-1}{\nabla^{2}} \rho_{a}^{\prime}=\frac{1}{8 \pi} \int d \vec{r} d \vec{r}^{\prime} \frac{\rho_{a}^{\prime}(\vec{r}) \rho_{a}^{\prime}\left(\vec{r}^{\prime}\right)}{\left|\vec{r}^{\prime}-\vec{r}^{\prime \prime}\right|}
$$

To recapitulate, the solution for a given source $(2.8)$ is constructed by choosing a gauge function $U$, computing $\rho$ ' from (2.14); $\Phi$, from (2.13c); and finally, the potentials from (2.13a) and $(2.13 b)$ or $(2.15)$. When $(2.12)$ is met, one has solutions 
which in general are essentially time-dependent - a time-translation cannot be compensated by a gauge transformation. The only member of the family with gauge-artifactual time-dependence is the coulomb one where $U=I$. By continuously deforming $I$ to $\mathrm{U}$, one passes continuously from the static coulomb solution to its time-denendent generalization. 4

Our third solution is again static, but it differs from the coulomb one by the property that in the Abelian frame it does not vanish with the source; rather it becomes a pure gauge.

$$
\left.A^{\mu}\right|_{\substack{\text { zero } \\ \text { sivice }}}=\left(J^{-1} e^{\mu}{ }^{\mu}\right.
$$

[In the absence of sources, finite-energy solutions are necessarily trivial; ${ }^{6}$ thus the potentials either vanish or are pure gauges.] A closed expression for this solution has not been given; only a formula perturbative in the source is available. So that we can speak of orders of perturbation, we shall take the source to be $O(Q)$ where $Q$ is a convenient scale of magnitude for the source. [For example, $Q$ can be an overall factor.] This solution is most economically presented by first transforming out of the Abelian frame with the gauge function U, occurring in (2.17).

$$
\beta^{\prime}=U^{\prime} \rho U^{-1}=U \frac{\sigma^{3}}{2 i} U^{-1} q
$$

In the new frame, the vector potentials vanish with the source. Perturbative formulas for them are ${ }^{2}$ 


$$
\begin{aligned}
& A^{\prime}=\Phi+\Phi\left(Q^{3}\right) \\
& \vec{A}^{\prime}=\frac{1}{\nabla^{2}}[\Phi, \vec{\nabla} \Phi]+O\left(Q^{4}\right) \\
& \Phi=-\frac{1}{\nabla^{2}} \rho^{\prime}
\end{aligned}
$$

Primes remind that the quantities are displayed in a non-Abelian frame. The gauge function $U$ is not arbitrary but must be chosen so that the consistency condition (2.2) is saisfied. It is a consequence of that equation and of $(2.18 \mathrm{~b})$ that we must have

$$
\left[\mp, \nabla^{2} \Phi\right]=0
$$

The following is the temporal gauge equivalent to. (2.18).

$$
\begin{aligned}
& A^{\prime}=0 \\
& \vec{A}^{\prime}=\vec{\nabla}^{\prime} \Phi t+\left(\frac{1}{2} t^{2}+\frac{1}{\nabla^{2}}\right)[\Phi, \vec{\nabla} \Phi]+Q\left(Q^{3}\right)
\end{aligned}
$$

The electric field is $O(Q)$,

$$
\vec{E}=-\vec{\nabla} \Phi-t[\Phi, \vec{\nabla} \Phi]+C\left(Q^{3}\right)
$$

and the magnetic field is $O\left(Q^{2}\right)$.

$$
\begin{aligned}
& \vec{B},=\vec{\nabla}^{-1} \times[\Phi, \vec{\nabla} \Phi]+O\left(Q^{3}\right) \\
& \vec{\nabla}^{-1}=\vec{\nabla} / \vec{\nabla}^{2}
\end{aligned}
$$

[The time dependence in $(2.20)$ is of course a consequence of the gauge choice, as comparison with (2.18) shows.] In the 
primed frame, the solution appears similar to the coulomb one (2.9) or (2.10), save that the non-vanishing commutator $[\Phi, \vec{\nabla} \Phi]$ prevents the expressions from closing. Hence we call the above a "non-Abelian Coulomb" solution to contrast it with the "Abelian Coulomb" discussed at the outset. The energy further exhibits similarities with the Abelian Coulomb case. The formula to lowest order in $Q$ follows from (2.3), (2.18d) and (2.20c).

$$
\begin{aligned}
& E^{0}=\frac{1}{2} \int \rho_{a}^{\prime} \frac{-1}{\nabla^{2}} \rho_{a}^{\prime}+O\left(Q^{4}\right) \\
& =\frac{1}{8 \pi} \int d \vec{r} d \vec{r}^{\prime} \frac{\rho_{a}^{\prime}\left(\vec{r}^{\prime}\right) \rho_{a}^{\prime}\left(\vec{r}^{\prime}\right)}{\left|\vec{r}^{\prime}-\vec{r}^{\prime}\right|}+C\left(Q^{4}\right)
\end{aligned}
$$

A specific example of a non-Abelian Coulomb solution is given when the source in the Abelian frame is spherically symmetric.

$$
\sigma_{a}=\sigma_{a 3} q(t)
$$

One then verifies that (2.19) is satisfied with the charge density in the radial frame;

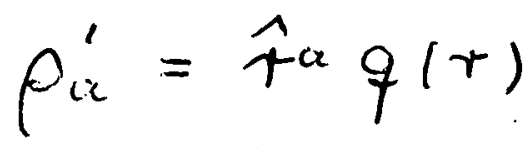

i.e. $U$ is the gauge transformation which rotates the third axis into the radial axis. A further interesting feature is that the present solution carries less energy than the corresponding coulomb one. 7 


$$
\varepsilon=\frac{1}{8 \pi} \int d \vec{r} d \vec{r}^{\prime} \frac{q(r) q\left(r^{\prime}\right)}{\left|\vec{r}^{\prime}-\vec{r}^{\prime}\right|} \hat{r} \cdot \hat{r}^{\prime}+O\left(Q^{4}\right)<\sum_{C}
$$

The fourth and last solution, that I mention in this subSection, generalizes the static non-Abelian Coulomb in a timedependent fashion, quite similarly to the way that the second solution, Eqs. (2.13), generalizes the Abelian Coulomb. It is constructed by choosing an arbitrary gauge transformation $U$ and transforming the source once again.

$$
\rho^{i \prime}=U \rho^{\prime} U^{-1}
$$

We use double primes to distinguish this source from $\rho$ - the source in Abelian frame - and from $\rho^{\prime}$ - the source in the gauge transformed frame where the non-Abelian Coulomb solution has a simple perturbative expansion, see (2.18) or (2.20). Next we take the regular solution of Poisson's equation

$$
\Phi=-\frac{1}{\nabla^{2}} \rho^{\prime \prime}
$$

and build the time-dependent solution perturbatively in $\Phi$, in the temporal gauge. 5

$$
\begin{gathered}
A^{\prime \prime} 0=0 \\
\vec{A}^{\prime \prime}=\vec{\nabla} \Phi t+\left(\frac{t^{2}}{2}+\frac{1}{\nabla^{2}}\right)\left([\Phi, \vec{\nabla} \Phi]-\vec{\nabla}^{-1}\left[\Phi, \nabla^{2} \Phi\right]\right) \\
+C^{(2.25 a)}
\end{gathered}
$$


One readily computes the electric field, which is $O(Q)$.

$$
\left.\vec{E}^{\prime \prime}=-\vec{\nabla} \Phi-t\left(\left[\Phi, \vec{\nabla}^{\prime} \Phi\right]-\vec{\nabla}^{-1}\left[\Phi, \nabla^{2} \Phi\right]\right)+\vec{C}^{-} \Phi^{3}\right)
$$

The $O\left(Q^{2}\right)$ magnetic field

$$
\vec{B}^{\prime \prime}=\vec{\nabla}^{-1} \times[\Phi, \vec{\nabla} \Phi]+C\left(C^{3}\right)
$$

is of the same form as in the static non-Abelian Coulomb solution, (2.20d) which is here included when $U=I$. Just as in the Abelian situation, by continuously changing $I$ to. $U$, we obtain a continuous deformation of the static non-Abelian Coulomb into its time-dependent generalization. In both cases the magnetic field retains the same form during the deformation. Once again, the $O\left(Q^{2}\right)$ energy is given by a coulombic formula, as follows from $(2.3),(2.24)$ and $(2.25 \mathrm{c})$.

$$
\begin{aligned}
& C=\frac{1}{2} \int P^{\prime \prime} \frac{-1}{\nabla^{2}} P_{i}^{\prime \prime}+O\left(Q Q^{4}\right) \\
& =\frac{1}{8 \pi} \int d \vec{r}^{\prime} d \vec{r}^{\prime} \frac{\rho_{u}^{\prime \prime}\left(\vec{r}^{\prime}\right) \rho_{a}^{\prime \prime}\left(\vec{r}^{\prime}\right)}{\left|\vec{r}^{\prime}-\vec{r}^{\prime \prime}\right|}+C\left(Q Q^{4}\right)
\end{aligned}
$$

The similarities between the four solutions should be apparent. Indeed, if for different gauge frames a common [unprimed notation is used, a master formula which presents all four may be given. 5 Define first the vector $\vec{C}$.

$$
\vec{C}=[\Phi, \vec{\nabla} \Phi]=\frac{-1}{\nabla^{2}} \rho
$$

Then, in the temporal gauge, set. 


$$
\begin{aligned}
& A^{0}=0 \\
& \vec{A}=-\frac{1}{\nabla^{2}}\left[\vec{\nabla} p \dot{t}+\left(\frac{1}{2} t^{2}+\frac{1}{\nabla^{2}}\right) \vec{\nabla} \times \vec{\nabla} \times \vec{C}\right]
\end{aligned}
$$

The static, Abelian Coulomb has vanishing $\vec{C}$, while vanishing $\vec{\nabla} \times \vec{C}$ leads to its time-dependent generalization as is seen from (2.12) and (2.13). Furthermore, according to (2.19) and (2.20), vanishing $\vec{\nabla} \cdot \vec{C}$ corresponds to the static, non-Abelian coulomb solution while its time-dependent generalization (2.25) has no restrictions on $\vec{C}$. For the first two solutions (2.28) is exact; for the last two it is accurate up to $O\left(Q^{2}\right)$. The $O\left(Q^{2}\right)$ formula

$$
C=\frac{1}{2} \int_{a} \frac{-1}{\nabla^{2}} \rho_{a}+O\left(C_{x}{ }^{4}\right)
$$

gives the complete energy for the exact solutions, and the $O\left(Q^{2}\right)$ contribution to the perturbative ones. For the two static solutions, the quantity in (2.29) is stationary against variations of $\rho_{a}$ which preserve its length [gauge transformations]. 8 The Coulomb solution is seen to maximize (2.29).

\section{$\ddot{C}$ Sources with "Critical strength}

When the source strength $Q$ increases, the previous solutions continue to be present. For the Abelian coulomb, and its time-dependent generalization, the closed expressions given 
above hold for arbitrary $Q$. For the non-Abelian Coulomb with its time-dependent generalization, one must calculate perturbatively terms higher order in ' $Q$; a tedious procedure with unknown convergence properties. Alternatively one can do numerical computations.

Furthermore as $Q$ increases, solutions appear which require a critical, minimal source strength to support them. Very little is known about these, and the numerical method is presently the only effective means of investigation, We review one such example. 2,9

When the source is radially symmetric, as in (2.22a), we have the spherically symmetric Abelian Coulomb solution. Also by passing to the radial frame (2.22b) we can exhibit the perturbative non-Abelian Coulomb solution. Bỳ iterating Eqs. (2.18) a few orders in $Q$, it is found that the form of the potentials remains within the following Ansatz.

$$
\begin{aligned}
& A^{0}=\frac{\hat{r} \cdot \vec{\sigma}}{2 i} \frac{1}{r} f\left(r / r_{0}\right) . \\
& \vec{A}=\frac{\hat{r} \times \vec{v}}{2 i} \frac{1}{r}\left[\alpha\left(r / r_{0}\right)-1\right]
\end{aligned}
$$

Here $r_{0}$ is a length scale. In this sub-section we shall always remain in the radial frame;

$$
f_{c i}=\frac{\hat{r}^{a}}{r_{0}^{3}} \text { of }\left(r / r_{0}\right)
$$

hence primes on the potentials are dropped. The above Ansatz 
is postulated for the complete static solution and the mode functions satisfy the following non-linear differential equations, which are all that remains of Gauss' and Ampère's laws.

$$
\begin{aligned}
& -f^{\prime \prime}+\frac{2 a^{2}}{x^{2}} f=x q \\
& -a^{\prime \prime}+\frac{a^{2}-1-f^{2}}{x^{2}} a=0
\end{aligned}
$$

All functions depend only on $x=r / r_{0}$, and the dash indicates differentiation with respect to that variable. More general radially symmetric Ansätze can be given, but it has been proven that static, radial solutions necessarily fall into the above restrictions. ${ }^{2}$ [We emphasize that the Abelian Coulomb solution does not lie within the Ansatz (2.30), and cannot be found in the solutions to (2.32); in the radial frame, the Abelian coulomb solution is not radially symmetric.] Requiring finiteness of the energy

$$
C=\frac{4 \pi}{r_{0}} \int_{0}^{\infty} d x\left[\left(a^{\prime}\right)^{2}+\frac{1}{2 x^{2}}\left(a^{2}-1\right)^{2}+\frac{1}{2}\left(f^{\prime}\right)^{2}+\frac{1}{x^{2}} f^{2} a^{2}\right]
$$

- the above is the form that (2.3) takes within the Ansatz (2.30) - imposes boundary conditions at the origin and at infinity. At the origin the potentials must vanish rapidly: $f(0)=0, a(0)=1, A^{0}(0)=0, \vec{A}(0)=0$. At infinity two types of behavior are allowed: type $I$, where the potentials vanish as in the origin; type II, where the vector potential tends to a nontrivial pure gauge, $a(\infty)=-1, \underset{\mathrm{A}}{\vec{r} \rightarrow \infty} \underset{i}{i} \frac{\hat{r} \times \vec{\sigma}}{r}=-(i \vec{\sigma} \cdot \hat{r}) \vec{\nabla}(-i \sigma \cdot \hat{r})$. The 
type I solution is the previously perturbatively encountered non-Abelian Coulomb $\left[a=1+O\left(Q^{2}\right)\right.$, hence a never equals. -1$]$. The type II is a new, non-perturbative solution.

Numerical computation confirms the above, with the further surprise that type II comes in two branches, once $Q$ exceeds a critical magnitude. 2 Hence we call this the "bifurcating" solution. Fig. I shows a plot of the energy versus source strength for solutions with a delta-shell charge density.

$$
\rho_{a}=\frac{\hat{\gamma}^{a}}{\gamma_{0}^{2}} Q d\left(r-r_{0}\right)
$$

The Coulomb parabola [which does not lie within the Ansatz] is also exhibited for comparison. Note that the non-Abelian Coulomb [type I] carries lower energy than the Abelian Coulomb for all Q, even outside the perturbative regime. The bifurcation point where the two type II solutions first occur is found numerically to be $Q=5.286$. In Figs. 2,3 and 4 the mode functions $f$ and a are displayed for the various solutions.

III. STABILITY IN DYNAMICAL SYSTEMS

A. Review of the General Theory

We consider a time-translation invariant system whose equations of motion for the $2 \mathrm{~N}$ dynamical variables $P_{n}$ and $Q_{n}$ ' $n=1, \ldots, N$, can be obtained from a Hamiltonian $H(P, Q)$, which is also the conserved energy $\mathcal{C}$. 


$$
\begin{aligned}
& \dot{P}_{n}=-\frac{\partial H(P, Q)}{\partial Q_{n}} \\
& \dot{Q}_{n}=\frac{\partial H(P, Q)}{\partial P_{n}}
\end{aligned}
$$

A static solution, one for which $\dot{P}$ and $\dot{Q}$ vanish, is a critical point of $\mathrm{H}$, and vice versa, stationary points of the energy define static solutions. [An over-dot means differentiation with respect to time.]

We wish to ascertain whether a static $\operatorname{solution}\left\{P^{(s)}, Q^{(s)}\right\}$ is stable. "Stable" by definition will mean the following: Take a configuration of the form $\left\{P^{(s)}+\delta P, Q^{(s)}+\delta Q\right\}$, substitute in $(3.1)$ and Iinearize about $\left\{P^{(s)}, Q^{(s)}\right\}$ to obtain Iinear equations for the fluctuating quantities $\{\delta P, \delta Q\}$. When the Iinear equations produce exponential growth in time for the fluctuations, the solution is unstable; otherwise, it is stable. In other words, for stable motion the small quantities $\{\delta P, \delta Q\}$ fluctuate harmonically in time with real frequency, while complex frequencies signal instability.

The above criterion for stability is also in accord with quantum-mechanical ideas. The first quantum correction to the energy of a state involves the fluctuation frequencies. That quantity must be real for the state to be quantum-mechanically stable.

Note that growth in time of the fluctuations smaller than exponential, say polynomial, is not a sign of instability. In such a circumstance, the eigenfrequencies are degenerate, but 
$-18-$

still real, and the quintal energy remains real.

An intuitively appealing idea is that stability should be connected with minimizing the energy: the static solution which is a stationary point should also be a [local] minimal point.

More precisely, the minimality condition is the requirement that only non-negative eigenvalues occur for the quadratic Hamiltonian matrix, $\mathscr{H}$, defined by expanding $H(P, Q)$ about $\left\{\mathrm{P}^{(s)}, \mathrm{Q}{ }^{(s)}\right\}$ and retaining quadratic terms in $\{\delta \mathrm{P}, \delta Q\}$. [Linear terms vanish since the expansion is about a critical point.]

$$
\begin{aligned}
& H(P, Q)= H\left(P^{(s)}, Q^{(s)}\right)+ \\
& \frac{1}{2} \delta P_{n} T_{n m} \delta P_{m}+\delta P_{n} G_{n m} \delta Q_{m}+\frac{1}{2} \delta Q_{n} V_{n m} \delta Q_{m} \\
&+\ldots \\
&=H\left(P^{(s)}, Q^{(s)}\right)+\frac{1}{2} \widetilde{X} \mathscr{J} \mathcal{X} X+\ldots \\
& \mathcal{H}=\left(\begin{array}{cc}
T & G .2) \\
\widetilde{G} & V
\end{array}\right) \quad X=\left(\begin{array}{c}
\delta P \\
\delta Q
\end{array}\right)
\end{aligned}
$$

[The tilde indicates transposition.] The minimality condition demands

$$
\operatorname{det}(\mathscr{O H}-\lambda I)=0 \Rightarrow \lambda \geqslant 0
$$

In fact minimality is a sufficient condition for stability

- a result, known as Dirichelet's theorem, which will become apparent below - but by no means is it a necessity. ${ }^{10}$ There are indeed familiar physical systems [tops, gyroscopes, planetory configurations] which are stable, even though their energy 
is not locally minimal. To derive a more general criterion we expand (3.1) around the static solution and find

$$
\begin{aligned}
& \operatorname{gf} X=i \eta \dot{X} \\
& \eta=\left(\begin{array}{cc}
0 & -i I \\
i I & 0
\end{array}\right)
\end{aligned}
$$

By making a monochromatic Ansatz for $\mathrm{x}$,

$$
X=e^{-i \omega t} x
$$

we recognize the [constant] $x$ as simplectic eigenvectors of H with simplectic eigenvalue $\omega$.

$$
g f_{x}=\omega \eta x
$$

It is clear that our definition of stability requires the w's be real; this is known as Liapunov's theorem. 10

$$
\operatorname{det}(g e-\omega \eta)=0 \Rightarrow \omega \text { wal }
$$

The point is that (3.9) is in general different from (3.4) and can be satisfied when (3.4) fails.

If $(3.8)$ is premultiplied by $x^{\dagger}$, where the dagger indicates transposition and complex conjugation,

$$
x^{\dagger} \mathscr{H} x=\omega x^{\dagger} \eta x
$$

we see that the left-hand side is real, being real symmetric, hence Hermitian. Also $x^{\dagger} \eta x$ is real since $\eta$ is Hermitian, and we conclude that $\omega$ can fail to be real only when $x$ to $x$ and 
$x^{\dagger} n x$ vanish. So when $\mathscr{H}$ is positive definite, $\omega$ is real and Dirichelet's theorem is established: minimality implies stability. But of course $\omega$ may be real without $\mathscr{H}$ being positive definite.

One may consider $n$ as a metric in the vector space of the $x^{\prime} s$. Then $(3.8)$ is the condition that $x^{\dagger} \mathscr{L} x$ be stationary against variations of $x$ which preserve the simplectic length $x^{\dagger} n x$. Instability can occur only when there are zero length simplectic eigenvectors of $\mathscr{H}$. The eigenvalue equation in (3.9) is relevant to the program of diagonalizing of by simplectic matrices, just as the corresponding equation in (3.4) arises when diagonalizing with orthogonal matrices. [A matrix $M$ is simplectic when $\tilde{M} n M=\eta \cdot]$

The conditions (3.4) and (3.9) are clearly different, and no simple relationship exists between the two in the general case. In practice, we can specialize somewaht. Firstly, the kinetic energy matrix $\mathrm{T}$ in (3.2) and (3.3) is taken to be positive definite; with an appropriate definition of coordinates, we may choose it to be the identity. Secondly, the offdiagonal matrix $G$ arising from mixed $p-q$ terms in the Hamiltonian, which are frequently called gyroscopic or coriolis terms, is always anti-symmetric, when the theory is derivable from a Lagrangian. The reason is that any symmetric piece in such velocity dependent forces corresponds to a total time-derivative in the Lagrangian and may be dropped. Thus we are led to a simpler form for Ke. 


$$
\begin{gathered}
\mathscr{f f}=\left(\begin{array}{cc}
I & G \\
-G & V
\end{array}\right) \\
\widetilde{G}=-G, \quad \tilde{V}=V
\end{gathered}
$$

with this $\mathscr{H}$, the minimality condition $(3.4)$ reduces to

$$
\operatorname{ctet}(-\tilde{G} G+(1-\lambda)(V-\lambda I))=0 \Rightarrow \lambda \geqslant 0
$$

while the stability condition becomes

$$
\operatorname{det}\left(-\tilde{G} G-2 i \omega G+V-\omega^{2} I\right)=0 \Rightarrow \omega \text { real }
$$

When gyroscopic forces are absent, the two conditions coincide and $\omega^{2}$ may be identified with $\lambda$. In that case instability occurs only for imaginary $\omega$. In the presence of gyroscopic terms, there exist stable static solutions which do not minimize the energy, while instability can exist with complex $\omega$. When is as in (3.11), the condition for instability, $x^{\dagger} n x=0$, is equivalent to

$$
R_{e} \omega=i \frac{\delta Q_{n}^{*} G_{n m} \delta Q_{m}}{\delta Q_{n}^{*} \delta Q_{n}}
$$

Whe shall use the phrase "gyroscopic stability" when we wish to distinguish this form of stability from the more familiar "energetic stability". A hint for gyroscopic stability occurs when we can find arbitrarily close to a static solution harmonic fluctuations that lower the energy. As we shall show, such con- 
figurations exist in the Yang-Mills theory. Instability would be indicated when there are, arbitrarily close to the static solution, time-dependent solutions which decrease the energy and grow exponentially in time.

To conclude this review of stability theory, let us remark that although we discussed. Dirichelet's sufficient condition in terms of the energy constant of motion, a similar criterion can be formulated by reference to other constants of motion. This generalization is useful when analyzing solutions invariant with respect to the symmetry transformation which is associated with the constant in question. 10 .

\section{B. Stability Analysis for Yang-Mills Theory}

We turn now to the stability analysis of static solutions for the Yang-Mills equations; but before we use the ideas sketched above, we must recognize that there are two ways in which the Yang-Mills field theory differs from the simple Familtonian. Firstly, rather than 2N degrees of freedom, there are an infinite number. This causes matrices to be replaced by differential operators, summations by integrations, etc., thus raising questions of convergence and uniformity. We shall not concern ourselves with this complication, even though there will be occasion to refer to it in the course of our development. Secondly, the Hamiltonian formulation now has constraints. This has already been dealt with in section II. Here we observe that the small fluctuation equations, which follow from (2.6) by linear- 
$-23-$

icing around a static solution $A_{a}^{\mu}$, are

$$
\begin{aligned}
O=\vec{D}_{a b} \cdot \delta \vec{E}_{b} & +\varepsilon_{a b c} \vec{E}_{b} \cdot \delta \vec{A}_{c} \\
\mathscr{Z}_{a b}^{0} \delta \vec{E}_{b}= & \vec{D}_{a b} \times \delta \vec{B}_{b}+\varepsilon_{a b c} \vec{E}_{b} \delta A_{c}^{0} \\
& -\varepsilon_{a b c} \vec{B}_{b} \times \delta \vec{A}_{c} \\
\delta \vec{E}_{a}= & -D_{a b}^{0} \delta \vec{A}_{b}-\vec{D}_{a b} \delta A_{b}^{0}
\end{aligned}
$$

$(3.15 a)$

$(3.15 b)$

$(3.15 c)$

$\delta \vec{B}_{a}$ is shorthand for $\vec{\sigma}_{a b} \times \delta A_{b}$. The quadratic energy, obtained by expanding (2.3) around the static solution, and using the Gauss' Jaw constraint for the fluctuations,

$$
\begin{aligned}
\delta^{(2)}=\frac{1}{2} \int d \vec{r} & \left\{\left(\delta \vec{E}_{a}\right)^{2}+2 \delta E_{a}^{i}\left(\varepsilon_{a c b} A_{c}^{0}\right) \delta A_{b}^{i}\right. \\
& +\left(\delta \vec{B}_{a}\right)^{2}-\delta A_{a}^{i}\left(\varepsilon^{i k j} \varepsilon_{a c b} B_{c}^{b}\right) \delta A_{b}^{j}
\end{aligned}
$$

is precisely of the form (3.11). In particular, an anti-symmetric gyroscopic term is present.

$$
G=\delta^{i j} \delta\left(\vec{r}-\vec{r}^{\prime}\right) \varepsilon_{a b c} A_{c}^{0}
$$

Eqs. (3.15) can also be obtained by taking (3.16) to be the quadratic Hamiltonian, and varying it subject to the constraint (3.15a) which is implemented with the help of a Lagrange multiplier $\delta A_{a}^{0}$. In other words, unconstrained variations are performed 
on

$$
\bar{Q}^{(2)}=\mathcal{E}^{(2)}-\int d \vec{r} \delta A_{a}^{0}\left(\vec{D}_{a b} \delta \vec{E}_{b}+\varepsilon_{a b c} \vec{E}_{b} \cdot \delta \vec{A}_{c}\right)
$$

and Eqs. (3.15a), (3.15b) and (3.15c) emerge respectively as

$$
\begin{aligned}
0 & =-\frac{\delta \bar{\varepsilon}^{(2)}}{\delta\left(\delta A_{a}^{c}\right)} \\
\partial_{t} \delta \vec{E}_{a} & =\frac{\delta \bar{\varepsilon}^{(2)}}{\delta\left(\delta \vec{A}_{a}\right)} \\
\partial_{t} \delta \vec{A}_{a} & =-\frac{\delta \bar{\varepsilon}^{(2)}}{\delta\left(\delta \vec{E}_{a}\right)}
\end{aligned}
$$

With a monochromatic Ansatz for the time-dependence, the above take on the form of a simplectic eigenvalue problem, equivalent to stationarizing $\bar{\varepsilon}(2)$, subject to the constraint that the simplectic length of $\left(\begin{array}{r}-\delta \vec{E}_{a} \\ \delta \vec{A}_{a}\end{array}\right)$ be fixed, and subject to the constraint of Gauss' law for the fluctuations. In short, the YangMills model is seen to fit the general theory quite nicely. Before making use of these equations to analyze stability, we comment on their properties. An integrability condition follows from (3.15b). By taking the covariant divergence, one finds that the infinitesimal version of (2.2) must be satisfied.

$$
\varepsilon_{a b c} \delta A_{b}^{0} p_{c}=0
$$

Also vice versa: $(3.20)$ and the integrability condition on (3.15b) imply, together with $(3.15 \mathrm{c})$, that the covariant time- 
derivative of the right-hand side in (3.15a) vanishes.

Eqs. (3.15) possess a local gauge invariance.

$$
\begin{aligned}
& \delta \vec{E}_{a} \rightarrow \delta \vec{E}_{a}-\varepsilon_{a b c} \vec{E}_{b} \theta_{c} \\
& \delta A_{a}^{0} \rightarrow \delta A_{a}^{0}-\partial_{t} \theta_{a}-\varepsilon_{a b} A_{b}^{0} \theta_{c} \\
& \delta \vec{A}_{a} \rightarrow \delta \vec{A}_{a}+\vec{\nabla} \theta_{a}-\varepsilon_{u b c} \vec{A}_{b} \theta_{c}
\end{aligned}
$$

Here $\theta_{a}$ is a local function which must be parallel to the source.

$$
\sum_{a b c} \theta_{b} \rho_{c}=0
$$

[There is also a gauge covariance: a gauge transformation on the background fields is compensated by a homogeneous gauge transformation on the fluctuating quantities. We shall not make use of this property.]

It. is clear from (3.20) and (3.22) that the external charge density defines a direction in group space which we can call the "electromagnetic" direction, while the orthogonal directions can be termed "charged". Thus $A_{a}^{\circ}, \delta A_{a}^{\circ}, \theta_{a}$ and $\rho_{a}$ all lie in the electromagnetic direction and vanish in the charged direction. This reduces the allowed gauge transformations, in that the last term in (3.21b) must vanish. Observe also that the gyroscopic term (3.17) affects only the charged direction; the electromagnetic fluctuations are free of gyroscopic terms. 
It is possible to derive a gauge invariant fluctuation equation in the following way. The quantity

$$
\begin{aligned}
\vec{e}_{a} & =\delta \vec{E}_{a}+\varepsilon_{a b c} A_{b}^{0} \delta \vec{A}_{c} \\
& =-\partial_{t} \delta \vec{A}_{a}-\vec{D}_{a b} \delta A_{b}^{0}
\end{aligned}
$$

is gauge invariant with respect to the gauge transformations (3.21). Then by taking a covariant time-derivative of (3.15b) we arrive after some steps at

$$
\begin{aligned}
& \mathscr{D}_{a b}^{0} \mathscr{D}_{b c}^{c} \vec{e}_{c}+\vec{D}_{a b} \times{\overrightarrow{D_{b c}}}^{0} \vec{e}_{c}-\varepsilon_{a b c} \vec{B}_{b} \times \vec{e}_{c} \\
& =\mathscr{D}_{a b}^{0} \mathscr{D}_{b i}^{c} \vec{e}_{c}-\vec{D}_{a b} \cdot \overrightarrow{\mathscr{D}}_{b c} \overrightarrow{e_{c}}+\overrightarrow{\mathscr{D}_{a b}} \overrightarrow{\mathscr{\theta}}_{b c} \cdot \overrightarrow{e_{c}} \\
& =\bigcirc
\end{aligned}
$$

[This is most readily obtained in the $\delta A^{\circ}=0$ gauge, which can always be achieved with the transformation $(3.21 \mathrm{~b})$.]

Eq. (3.24) is gauge invariant and involves the unconstrained variable $\vec{e}_{a}$. It is remarkable that such an equation can be derived; the possibility to do so is intimately linked with the existence of an external charge density which defines a direction with respect to which the small fluctuations are constrained by $(3.20) .5$

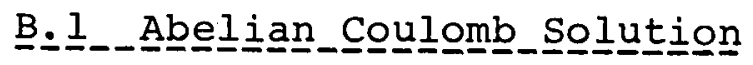

For the Abelian Coulomb solution, the general stability theory is easily applied. The small oscillation equations are best presented by introducing complex quantities in the charged 
direction.

$$
\begin{aligned}
& \delta \vec{E}=\frac{1}{\sqrt{2}}\left(\vec{E}_{1}+i \vec{E}_{2}\right) \\
& \delta \vec{A}=\frac{1}{\sqrt{2}}\left(\vec{A}_{1}+i \vec{A}_{2}\right) \\
& \vec{e}=\frac{1}{\sqrt{2}}\left(\vec{e}_{1}+i \overrightarrow{e_{2}}\right)
\end{aligned}
$$

Eq. (3.24) in the electromagnetic direction decouples completely,

$$
\partial_{t}^{2} \vec{e}_{3}+\vec{\nabla} \times \vec{\nabla} \times \vec{e}_{3}=0
$$

while in the charged direction we have simply

$$
\begin{aligned}
\left(\partial_{t}+i \varphi\right)^{2} \vec{e}+\vec{\nabla} \times \vec{\nabla} \times \vec{e} & =0 \\
\nabla^{2} \varphi & =-q
\end{aligned}
$$

The electromagnetic fluctuations are free; the charged ones describe the motion of charged vector mesons in an external electric field with a potential $\phi .{ }^{11}$

Detailed analysis of the equations can be performed in frequency space. Note that the electromagnetic equation involves $\omega^{2}$ as an eigenvalue of a Hermitian operator, hence it is real. Only the issue remains whether $\omega^{2}$ is positive or negative. In the charged equation there appears $(\omega-\phi)^{2}$ and $\omega^{2}$ can be complex; it is not related to the eigenvalue of a Hermitian operator. This difference reflects the fact previously remarked upon: in the electromagnetic direction there are no gyroscopic terms, hence stability is equivalent to minimality. In the charged direction, the gyroscopic terms are 
present; they are responsible for the more complicated equation.

The electromagnetic fluctuations are obviously stable. Those in the charged directions are. stable in the absence of the external potential and by continuity they remain stable for a sufficiently small external potential. 3 As the external charge density increases in strength, an instability is expected to appear. This is not the instability of the KleinGordon equation in an $1 / r$ [Coulomb] potential, which has previously been remarked upon, and which is a consequence of the [presumably unphysical] singularity at the origin. ${ }^{12}$ In our examples the potentials are non-singular. Instead it is the instability of the Klein-Gordon equation in a strong external field. 13

Explicit computation with the delta-shell source, $q=\frac{\mathrm{Q}}{r_{0}^{2}} \delta\left(r-r_{0}\right)$, confirms the above remarks; instability sets in at $Q=$.

In spite of stability for weak sources, we expect as a consequence of the gyroscopic terms to find modes which, though harmonic, lower the energy. These can be readily exhibited, without passing to frequency space. We remain with the firstorder equations (3.15), and seek a solution with $\delta \vec{B} a=0$. In that case the charged portions of (3.15), with an Abelian Coulomb solution as the background field, reduce to

$$
\begin{aligned}
& 0=\vec{\nabla} \cdot \delta \vec{E}-i \delta \vec{A} \cdot \vec{\nabla} \varphi \\
& O=\left(\partial_{t}+i \varphi\right) \delta \vec{E}
\end{aligned}
$$


$-29-$

$$
\begin{aligned}
& \delta \vec{E}=-\left(\partial_{t}+i \varphi\right) \delta \vec{A} \\
& \delta \vec{B}=\vec{\nabla} \times \delta \vec{A}=0
\end{aligned}
$$

$(3.28 d)$

The solution of $(3.28 \mathrm{~b})$ and $(3.28 \mathrm{c})$ is

$$
\begin{aligned}
& \delta \vec{A}=\left[\vec{a}_{0}(\vec{r})+t \vec{a}_{1}(\vec{r})\right] \exp -i t \varphi(\vec{r}) \\
& \delta \vec{E}=-\vec{a}_{1}(\vec{r}) \text { exp -it } \varphi(\vec{r})
\end{aligned}
$$

and to satisfy $(3.28 a)$ and $(3.28 d)$ we must have

$$
\begin{aligned}
& \vec{a}_{0}=\vec{\nabla} \theta \\
& \vec{a}_{1}=-i \theta \vec{\nabla} \varphi-i \vec{\nabla}^{-1}(\theta q)
\end{aligned}
$$

(3. 29d)

where $\theta$ is an arbitrary function. Finally there is one more condition: $\vec{\nabla}^{-1}(\theta q)$ must be parallel to $\vec{\nabla} \phi$, which can be easily achieved, for example by setting $\theta q=\nabla^{2} F(\phi)$, where $F$ is arbitray. Thus equations (3.28) can be satisfied in terms of one function.

The quadratic energy (3.16) is seen to be negative. 14

$$
\begin{aligned}
\mathbb{C}^{(2)} & =\int\left[(q \theta) \frac{-1}{\nabla^{2}}\left(q \theta^{*}\right)-|\theta|^{2}(q)-\frac{1}{\nabla^{2}}(q)\right] \\
& =-\frac{1}{8 \pi} \int d \vec{r} d \vec{r}^{\prime} \frac{q(\vec{r}) q\left(\vec{r}^{\prime}\right)}{\left|\vec{r}^{\prime}-\vec{r}^{\prime}\right|}\left|\theta\left(\vec{r}^{\prime}\right)-\theta\left(\vec{r}^{\prime}\right)\right|^{2}
\end{aligned}
$$

(3.30) 
This rather peculiar fluctuation gives evidence that the coulomb solution is gyroscopicaliy stable since the energy decreases below its Coulomb value. ${ }^{5}$ Note from (3.29), the linear growth of the fluctuation with time, which however does not produce instability. Because the frequency is position dependent, it is not clear how to locate this mode among superpositions of functions with definite frequency. Nevertheless we have encountered it already! It is merely the time-dependent generalization of the Abelian Coulomb solution, Eqs. (2.12)-(2.16), when the latter is brought arbitrarily close to the static solution. ${ }^{15}$ The energy formula (3.30) can now be recognized as the $O\left(\theta^{2}\right)$ contribution to (2.16), when the source $\rho^{\prime}$ is a gauge transformation with gauge function $\theta$ of $\rho$, the source in the standard frame.

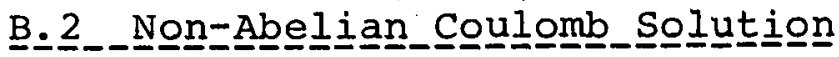

The non-Abelian Coulomb solution, Eqs. (2.18), follows in many respects, at least for weak sources, the behavior of the Abelian Coulomb solution. [Only the weak source regime is amenable to analytic treatment, since our formulas are given by a source strength power series.] The stability equations are now highly coupled, and have not been solved. However, by continuity with the sourceless problem one expects stability for weak sources. ${ }^{3}$ Moreover, one can show that this again must be an instance of gyroscopic stability, since the energy is lowered by the time-dependent generalization presented in Eqs. (2.24)(2.26), which can be taken arbitrarily close to the non-Abelian 
Coulomb solution. This is achieved by making $\rho "$, the source for the time-dependent solution, to be an infinitesimal gauge transformation of $\rho^{\prime}$, the source in the non-Abelian coulomb solution. The energy can then be computed from (2.26). The details are the following. Set

$$
f_{c}^{\prime \prime}=\left(1-\frac{i}{2} \theta_{b} \theta_{b}\right) \rho_{a}^{\prime}-\varepsilon_{a b c} \theta_{b} \rho_{c}^{\prime}
$$

This assures that $\rho_{a}^{\prime \prime}$ is a gauge transformation of $\rho_{a}^{\prime}$ taken through second order in $\theta_{a}$, which lies only in the charged direction.

$$
\theta_{a}(\vec{r}) \rho_{a}^{\prime}(\vec{r})=0
$$

It now follows from (2.26) that the $O\left(Q^{2}\right)$ energy is

$$
\begin{aligned}
& \zeta=\frac{1}{8 \pi} \int d \vec{r} d \vec{r}^{\prime} \frac{\rho_{a}^{\prime}\left(\vec{r}^{\prime}\right) \rho_{u}^{\prime}\left(\vec{r}^{\prime \prime}\right)}{\left|\vec{r}^{\prime}-\vec{r}^{\prime \prime}\right|} \\
& -\frac{1}{4 \pi} \int d \vec{r}^{\prime} d \vec{r}^{\prime} \frac{p_{a}^{\prime}(\vec{r}) \rho_{b}^{\prime}\left(\vec{r}^{\prime}\right)}{\vec{r}^{\prime}-\vec{r}^{\prime} \mid} \varepsilon_{a b c} \Theta_{c}\left(\vec{r}^{\prime}\right) \\
& -\frac{1}{16 \pi} \int d \vec{r}^{\prime}\left(\hat{\psi} \vec{r}^{\prime} \frac{\rho_{a}^{\prime}\left(\vec{r}^{\prime}\right) \rho_{a}^{\prime}\left(\vec{r}^{\prime}\right)}{\left|\vec{r}^{\prime}-\vec{r}^{\prime}\right|}\left[\theta_{b}(\vec{r})-\theta_{b}\left(\vec{r}^{\prime}\right)\right]^{2}\right. \\
& -\frac{1}{8 \pi} \int d \vec{r} d \vec{r}^{\prime} \frac{\rho_{a}^{\prime}(\vec{r}) P_{i}^{\prime}\left(\vec{r}^{\prime}\right)}{\left|\vec{r}^{\prime}-\vec{r}^{\prime \prime}\right|} \theta_{a}\left(\vec{r}^{\prime}\right) \theta_{b}\left(\vec{r}^{\prime}\right) \\
& +O\left(Q^{4}\right)
\end{aligned}
$$

The first term is the $O\left(Q^{2}\right)$ non-Abelian Coulomb energy. The second may also be written as $\int d \vec{r} \Phi_{a}(\vec{r}) \varepsilon_{a b c} \nabla^{2} \Phi_{b}(\vec{r}) \theta_{c}(r)$, whence it is seen to vanish due to (2.19). The remaining two terms give the energy of the fluctuation. Unlike in (3.30), one cannot 
determine the sign by inspection, but after some straightforward manipulations, one can show that in the generic spherically symmetric case the terms are negative. 5

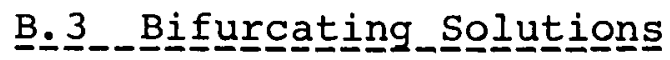

The bifurcating solutions, described in section IIC, exist only for sufficiently strong sources. Consequently, we have no closed-form expressions to analyze; yet precisely because there is a bifurcation, we can say a considerable amount without explicit computation. Consider first a solution to the static Yang-Mills equations for a definite source $p$ - Eqs. (2.6) with the left-hand side of $(2.6 b)$ and $(2.6 c)$ set to zero. Next imagine changing the source strength slightly, $\rho \rightarrow \rho+\delta \rho$, and looking for a new static solution. If the new solution is regularly related to the old one, the increments in the Yang-Mills fields will satisfy linear equations which are of the same form as the fluctuation equations (3.15), except that $\delta \rho$ occurs in the left-hand side of (3.15a) and time-derivatives are absent in $(3.15 b)$ and $(3.15 c)$. However, if we are at the bifurcation point, it must be impossible to solve these equations, and this happens if the homogeneous system has a non-trivial solution. In this way we arrive at the important observation that at the bifurcation point the stability equations have a zeroeigenvalue mode, and vice versa: a zero-eigenvalue mode indicates bifurcations, or generalizations thereof, in the static solutions viewed as functionals of the source. 
A zero eigenvalue mode in the stability equations does not necessarily signal the onset of instability. But let us assume that some of the stability equations can be manipulated into the form

$$
L \psi=\omega^{2} \psi
$$

where $L$ is a linear Hermitian operator, with a "potential" which is determined by the static Yang-Mills solution, itself a. function of source strength $Q$. At the critical value of $Q=Q_{C}$ there is a bifurcation, hence (3.34) indicates the existence of a "zero-energy bound state". As $Q$ increases beyond $Q_{C^{\prime}}$ we can expect that $\omega^{2}$ increases for one branch of the bifurcation, while for the other it decreases. We conclude therefore that when the stability equations follow the pattern here described, beyond the bifurcation point there is instability in one branch and stability can exist for the other.

We now show that the bifurcating Yang-Mills solution fits into the picture sketched above. In order to exhibit the instability, it is sufficient to consider just the radially symmetric sector of the theory; the sector in which the bifurcating solution was found.

For the radially symmetric source

$$
p_{\alpha}=\hat{r}^{a} q(r)
$$

the radially symmetric Ansatz is

$$
A_{a}^{0}=\hat{\gamma} \sim A^{0}
$$


$-34-$

$$
\begin{gathered}
A_{a}^{\hat{i}}=\varepsilon^{i a j} \frac{\hat{r} j}{r}\left(1+\varphi_{2}\right)+\frac{1}{r}\left(\delta^{i a}-\hat{r} i \hat{r} u\right) \varphi_{1} \\
+\hat{\gamma} i \hat{r} u A^{1}
\end{gathered}
$$

(3. 36b)

The functions depend on $r$ and $t$ and describe $a(1)$ Highs gauge model on a two-dimensional space with constant curvature. ${ }^{16}$ There is an Abelian gauge invariance.

The most general static solution is [gauge equivalent to] $\phi_{1}=0, A^{1}=0, \phi_{2}=-a, A^{0}=f / r$, where $a$ and $f$ are governed by (2.32). The time-dependent fluctuations

$$
\begin{aligned}
& A^{0}=A / r+\delta A^{0} \\
& A^{1}=\delta A^{1} \\
& \varphi_{1}=\delta \varphi_{1} \\
& \dot{\varphi}_{2}=-a+\delta \varphi_{2}
\end{aligned}
$$

satisfy

$$
\begin{aligned}
& \frac{\partial}{\partial r} e-\frac{4 a}{r} \delta \delta+2 a^{2} \delta A^{\circ}=0 \\
& -\partial_{t} e+2 a^{2} \delta A^{1}=0
\end{aligned}
$$

$(3.38 a)$

$$
\left(\partial_{t}^{2}-\frac{\partial^{2}}{\partial r^{2}}+\frac{3 a^{2}-1-f^{2}}{r^{2}}\right) \delta \varphi+\frac{2 a}{r} f \delta A^{0}=0
$$

We have used the gauge freedom to set $\delta \phi_{1}$ to zero, and have defined $\delta \phi_{2}$ to be $\delta \phi$. [In the gauge $\delta \phi_{1}=0, \delta \phi$ is a charge-neutral 
$-35-$

fluctuation.] Also the gauge-invariant fluctuating field strength e has been introduced.

$$
e=-r^{2}\left(\partial_{t} \delta A^{1}+\frac{\partial}{\partial r} \delta A^{0}\right)
$$

Finally by differentiating $(3.38 \mathrm{~b})$ with respect to time, and eliminating $\delta A^{l}$ with the help of (3.39), we may replace that equation by

$$
\left(\partial_{t}^{2}+\frac{2 a^{2}}{r^{2}}\right) e+2 a^{2} \frac{\partial}{\partial r} \delta A^{0}=0
$$

With a monochromatic Ansate, ERs. (3.38a), (3.38c) and (3.40) may be combined into the form (3.34), where

$$
\begin{gathered}
\psi=\left(\begin{array}{c}
\delta \varphi \\
e / 2 i a
\end{array}\right) \\
L=L^{+}=\left(\begin{array}{cc}
p^{2}+\frac{3 a^{2}-1-f^{2}}{r^{2}} & \frac{2 f}{r a} p a \\
a \frac{2 t}{r a} & p^{2}+\frac{a^{2}+1+f^{2}}{r^{2}}+2\left(\frac{a^{\prime}}{a}\right)^{2}
\end{array}\right) .
\end{gathered}
$$

Thus we see that for the charge-neutral fluctuations, the simplectic stability condition is reducible to a Hermitian problem. 
The bifurcation point, corresponding to a zero eigenvalue, is governed by equations obtainable also from (3.38) by setting the time derivatives to zero. Then it follows from (3.38b) that $\delta A^{1}$ vanishes, while the remaining two equations, reduce to a deformation of (2.32) with the identification $\delta a=-\delta \phi, \delta f=r \delta A^{\circ}$.

A numerical investigation of (3.34) and (3.41) is in progress. 5 We anticipate that the non-Abelian coulomb solution, which showed no bifurcations when the source strength was increased, will show no zero eigenvalue modes; $\omega^{2}$ will remain positive and that solution is always stable against radial deformation. [Non-radial deformations are not investigated.] The bifurcating solutions should lead to a zero-eigenvalue mode at the bifurcation point. Beyond it, the upper branch should show bound states in (3.34), hence be unstable. For the lower branch we anticipate no bound states and no radial instability.

The zero eigenvalue problem may be alternatively viewed as defining the bifurcation point. A crude analytic analysis for the delta-shell source (2.34) gives $Q_{C}=5.892$, in excellent agreement with the exact, numerically determined value of 5.826. This analysis will be presented elsewhere. 5

\section{CONCLUSION}

Finite-energy solutions to the Yang-Mills equations with arbitrary sources, can be studied perturbatively for weak sources. A rather comprehensive description is available. There exist at 
least two static solutions, the Abelian and non-Abelian Coulomb, with the latter carrying lower energy. They are accompanied by time-dependent generalizations which are continuous deformations of the static solutions. The time-dependent ones have the important property of lowering the energy, relative to the static configurations. The whole assembly of solutions can be compactly described in terms of the quantity $\vec{C}=[\Phi, \vec{\nabla} \Phi]$. Beyond the perturbative regime, it is difficult to study the problem analytically [save for the Abelian coulomb case], but numerical investigation does not expose any significant new structure.

Some questions remain. One would like to know how many different non-Abelian Coulomb solutions there are for a fixed source. [Thus far we have found only one.] Also one wonders whether there is a topological distinction between the Abelian and non-Abelian cases; a hint of one arises from the observation that the gauge transformation $U$, which takes the source from the Abelian frame to the non-Abelian frame in (2.18), is topologically non-trivial.

Solutions which are supported only by sources that exceed a critical strength, are known in isolated examples, but little of a general nature can be said about them at present. Presumably they are always characterized by bifurcations, and one wonders whether the bifurcating solutions are topologically different from the perturbative ones. Again one can point to a hint:at large distances, the radial non-Abelian Coulomb solution 
vanishes rapidly, while the bifurcating one tends to a non-trivial pure gauge. Also one would like to know how to characterize the different bifurcated branches.

The most interesting result of the stability analysis for Yang-Mills theory is the observation that both the Abelian and non-Abelian Coulomb solutions, when stable, are gyroscopically stable. In this way, the Yang-Mills model shares the physics of a top. 17 Modes which lower the energy, without introducing instability, have been identified. However, it is not clear how they are to be represented by superpositions of conventional monochromatic fluctuations.

Stability for weak sources can be established, but the behavior for strongex sources is thus far unknown, save for the Abelian coulomb case where an explicit formula allows for computations - the coulomb solution is unstable beyond a critical source strength. In the bifurcating solutions, the bifurcation point corresponds to a zero-eigenvalue mode in the stability equations, and one expects at least one of the bifurcated branches to be unstable.

While some further computations obviously suggest themselves, especially for strong sources, the most pressing open question concerns the relevance of these mathematical investigations to the quantum physics of Yang-Mills theory. 


\section{References}

1. P. Sikivie and N. Weiss, Phys. Rev. D (in press).

2. R. Jackiw, I. Jacobs and C. Rebbi, Phys. Rev. D (in press).

3. J. Mandula, Phys. Rev. DI4, 3497 (1976).

4. An example of this solution, with a radially symmetric source, $\rho_{a}=\delta_{a} q(r)$, is constructed by taking $\vec{A}_{a}=$ $-\vec{E}_{a} t+\delta_{a l} \vec{\nabla}\left(\alpha r^{2} \frac{d \phi}{d r}\right), \vec{E}_{a}=\frac{-\hat{r}}{r^{2}} \frac{1}{\alpha}\left\{\delta_{a 3} \sin \left(\alpha r^{2} \frac{d \phi}{d r}\right)+\delta_{a 2}\left[1-\cos \left(\alpha r^{2} \frac{d \phi}{d r}\right)\right]\right\}$, $\phi=-\frac{1}{\nabla} 2 q$. This is of the form (2.15) with $U=\exp \left(\frac{i \sigma^{1}}{2} \alpha r^{2} \frac{d \phi}{d r}\right)$. The energy is $=8 \pi \int_{0}^{\infty} \frac{d r}{\alpha^{2} r^{2}} \sin ^{2}\left(\frac{\alpha}{2} r^{2} \frac{d \phi}{d r}\right)$. Here $\alpha$ is an arbitrary parameter, which when set to zero gives the coulomb solution. This configuration is essentially the "total screening solution" found by P. Sikivie and N. Weiss, Phys. Rev. Lett. 40, 1411 (1978), and Phys. Rev. D18, 3809 (1978); except that they use a discrete parameter rather than our continuously varying $\alpha$. The feasibility of generalizing the total screening solution was pointed out by P. Pirilä and P. Presnajder, Nucl. Phys, B142, 229 (1978). The present formulation is given by Jackiw and Rossi, Ref. 5 .

5. R. Jackiw and P. Rossi (in preparation).

6. S. Coleman, in New Phenomena in Sub-Nuclear Physics, edited by A. Zichichi (Plenum, New York, 1977); S. Deser, Phys. Lett. $64 \mathrm{~B}, 463$ (1976).

7. The truth of this statement is manifest from (2.22c) for sufficiently small $Q$, so that the $O\left(Q^{4}\right)$ terms are negligible; and for charge densities $q(r)$ which never change sign, so that $q(r) q\left(r^{\prime}\right) \hat{r} \cdot \hat{r}^{\prime}<q(r) q\left(r^{\prime}\right)$. However, in Ref. 2 
it is shown that even for charge densities with varying signs the inequality in $(2.22 \mathrm{c})$ is valid, and that numerical computation at large $Q$ confirms the bound; see Fig. 1, below.

8. J. Goldstone (unpublished); see also Ref. 2.

9. Another example of a solution which exists when the source is of sufficient magnitude is Sikivie and Weiss' "magnetic dipole solution"; see Ref. 4. The source which supports this solution is studied by $Y$. Leroyer and A. Raychaudhuri, Phys. Rev. D (in press).

10. For an introduction to current, mainly mathematical research on stability of motion see C. Siegel and J. Moser, Lectures on Celestial Mechanics, (Springer Verlag, Berlin 1971). A simple discussion, referring to older physics research, is found in H. Jeffreys and B. Jeffreys, Methods of Mathematical Physics, (Cambridge University Press, Cambridge, 1972).

11. This form for the fluctuation equations in a coulomb background field was also given by M. Magg, Physics Letters $\underline{74 B}, 246$ (1978).

12. J. Mandula, Physics Letters 67B, 175 (1978); M. Magg, Physics Letters $74 \mathrm{~B}, 246$ (1978).

13. L. Schiff, H. Snyder and J. Weinberg, Phys. Rev. 57, 315 (1940); K. Johnson, Harvard Ph.D thesis (1954) (unpublished); A. Migdal, Zh. Eksp. Teor. Fiz. 61, 2209 (1972) [English translation: Soviet Physics JETP 34, 1184 (1972)]; A. Klein 
$-41-$

and J. Rafelski, Phys. Rev. DII, 300 (1975).

14. The truth of this statement is manifest for charge densities which do not change sign, so that $q(\vec{r}) q\left(\vec{r}^{\prime}\right)>0$. However, for spherically symmetric charge densities the proviso can be removed, see Ref. 15, below.

15. For spherically symmetric charge densities, we may take the solution described in Ref. 4. For small $\alpha$ it becomes an infinitesimal deformation of the Abelian Coulomb with energy $=2 \pi \int_{0}^{\infty} d r r^{2}\left(\phi^{\prime}\right)^{2}-\frac{\alpha^{2} \pi}{6} \int_{0}^{\infty} d r r^{6}\left(\phi^{\prime}\right)^{4}$, which is always less than the Coulomb energy, regardless of the sign of the source.

16. E. Witter, Phys. Rev. Lett. 38, 121 (1977).

17. That the physics of the top is encountered in the YangMills theory was previously remarked by J. Goldstone and R. Jackiw, Phys. Lett. $\underline{74 B}, 81$ (1978). Indeed it was in the context of the formalism developed in this paper that" some of the results summarized here were first encountered.

Reprints + Preprints Removed 


\section{FIGURE CAPTIONS}

All Figures are taken from Ref. 2.

Fig. I: Energy, in units of $2 \pi / r_{0}$ as a function of $Q$ for a delta-shell source of strength $Q$. The curve $C$ is the Abelian Coulomb parabola. The curve $I$ is the non-Abelian Coulomb solution. Curves IIa and IIb are the two branches of the bifurcating solution. The bifurcation point occurs at $Q=5.826$.

Fig. 2: Profiles of the functions $a$ and $f$ for the type I solution. Starting from the lowest curves the values of $Q$ in a delta-shell source are $1.41,2.53,4.04$ $6.43,10.05,14.18,19.93,23.38$, and 41.72 .

Fig. 3: Profiles of the functions $a$ and $f$ for the (a) (lower) branch of the type II bifurcating solution with a delta-shell source. $Q=5.86,6.44,8.09,11.05,15.71$, and 23.19. Correspondence between the individual curves and these values of $Q$ is established by the fact that, as $Q$ increases, so do $a^{\prime \prime}(0)$ and $f(l)$.

Fig. 4: Profiles of the functions $a$ and $f$ for the (b) (upper) branch of the type II bifurcating solution with a deltashell source. $Q=6.53,8.61,12.10,17.85,28.06$, and 49.16. For these curves, as $Q$ increases, a" (0) decreases and $f(1)$ increases. 


\section{$-43-$}

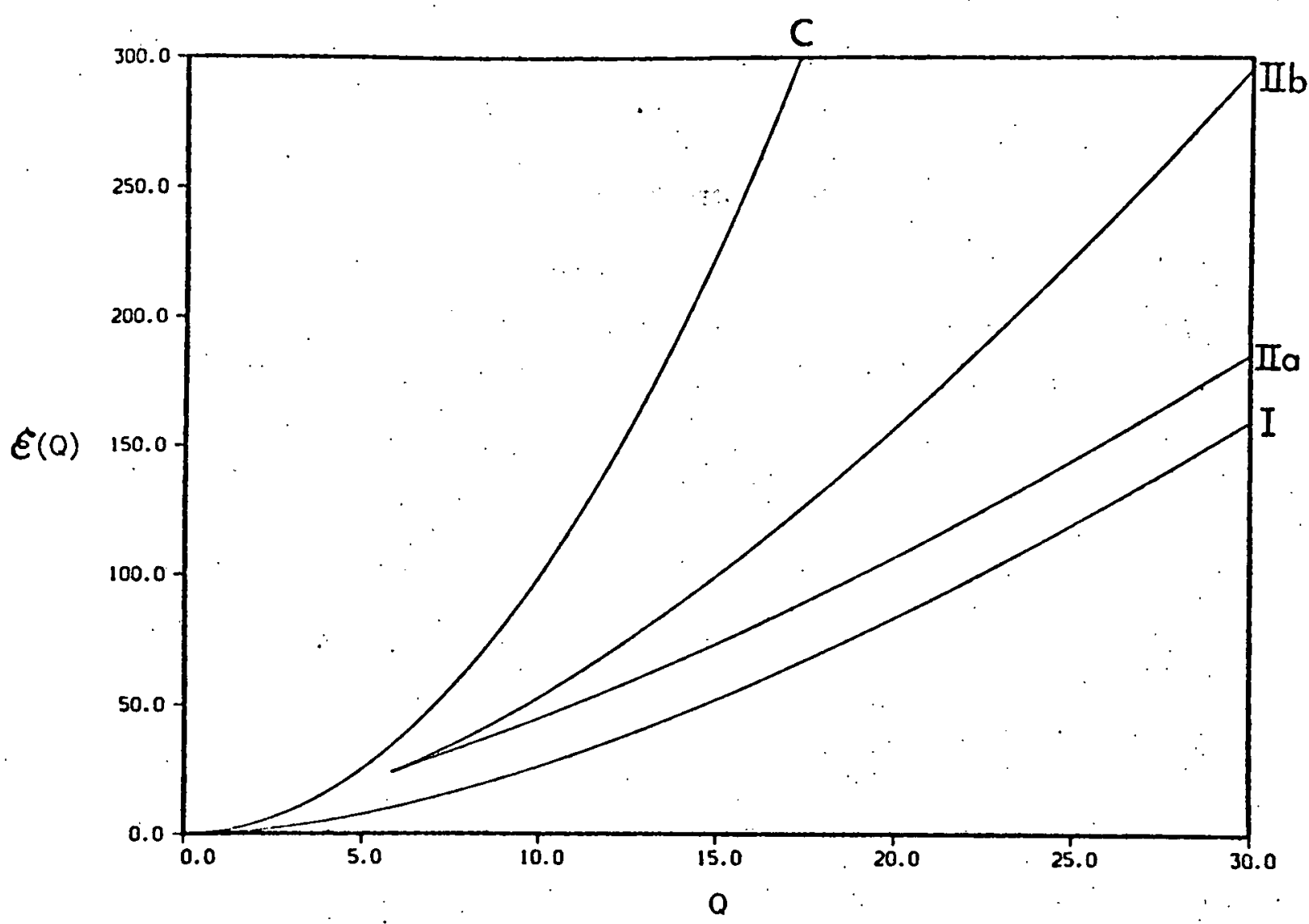

FIG 1 

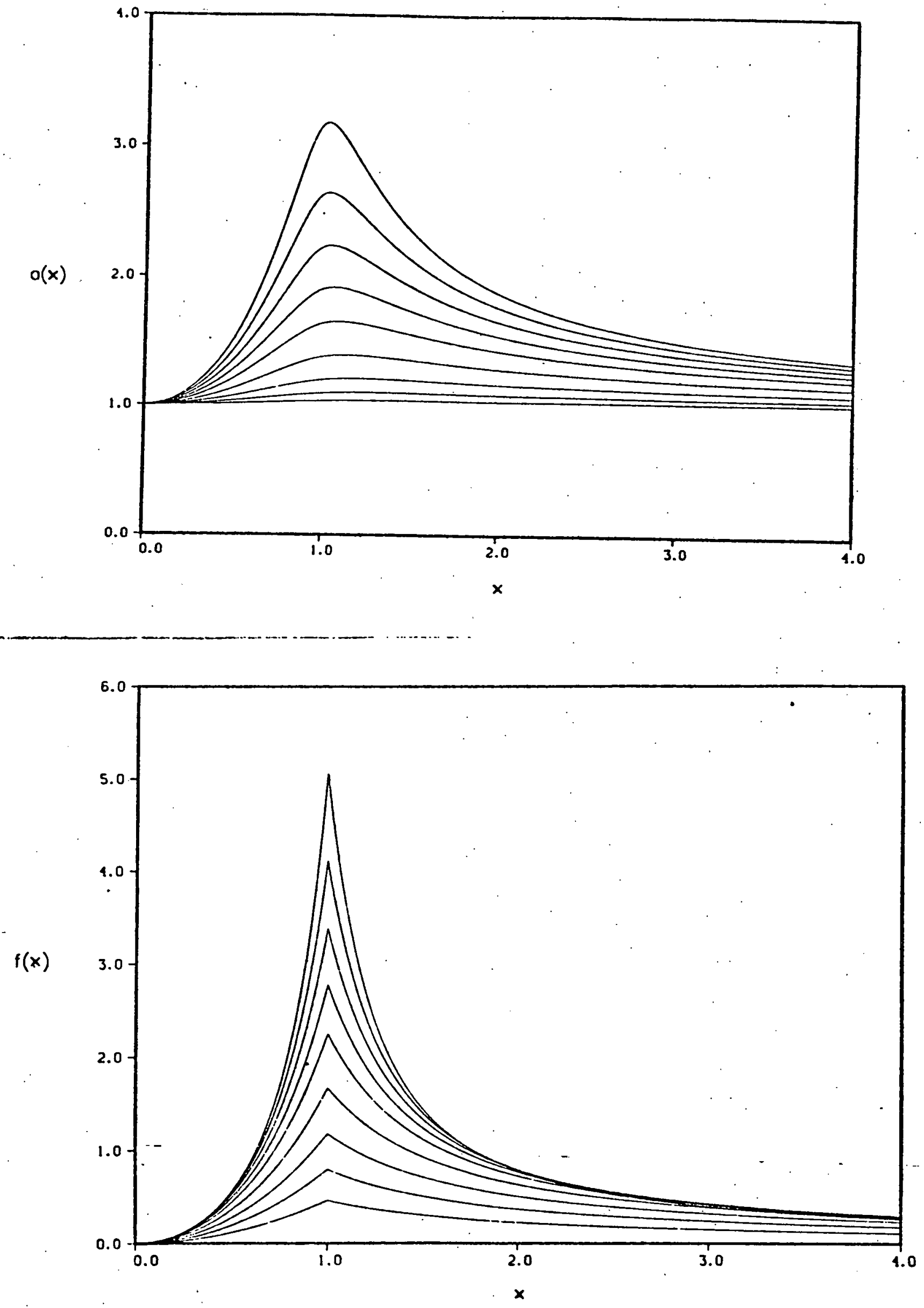

FIG 2 

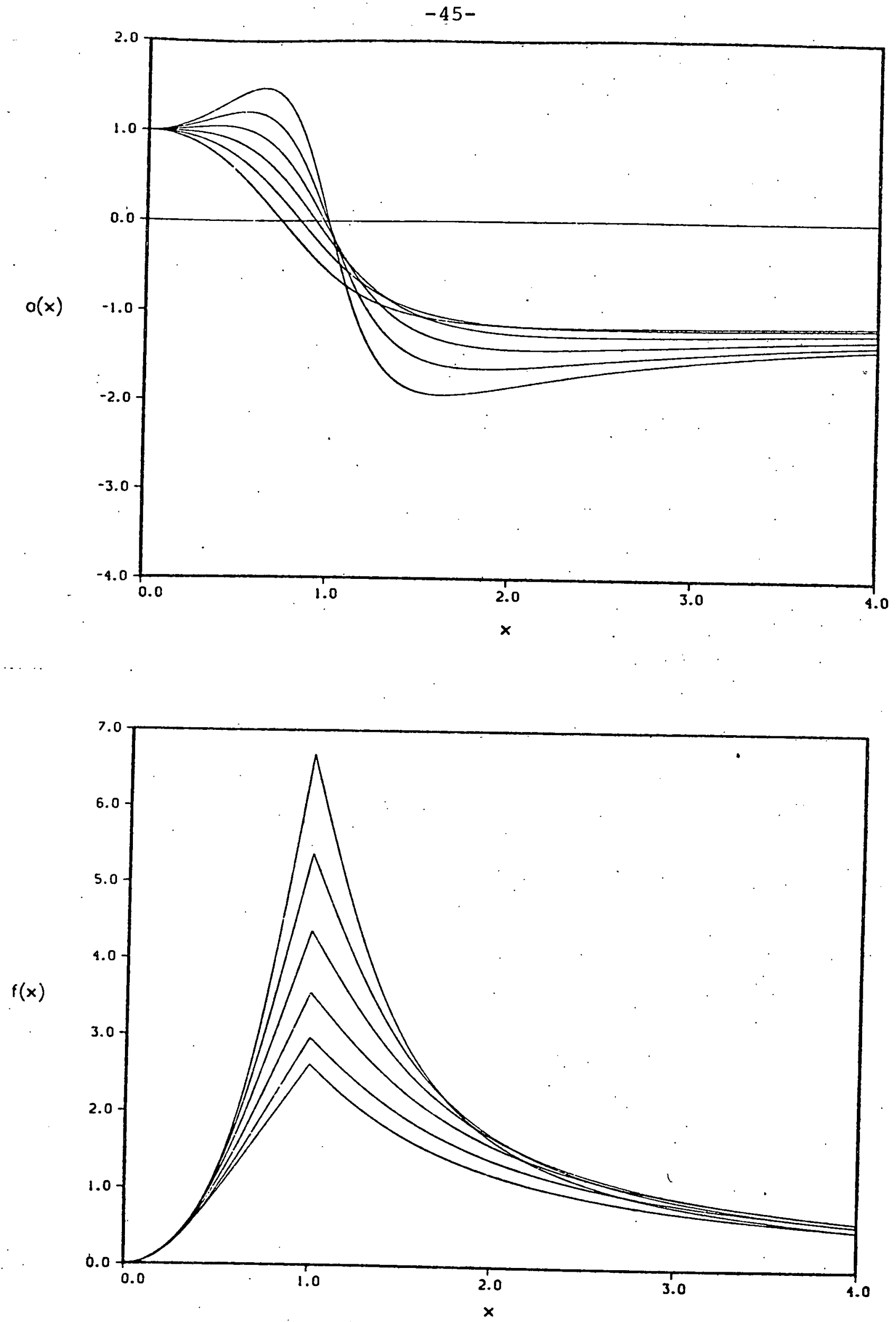

FIG 3 
$-46-$
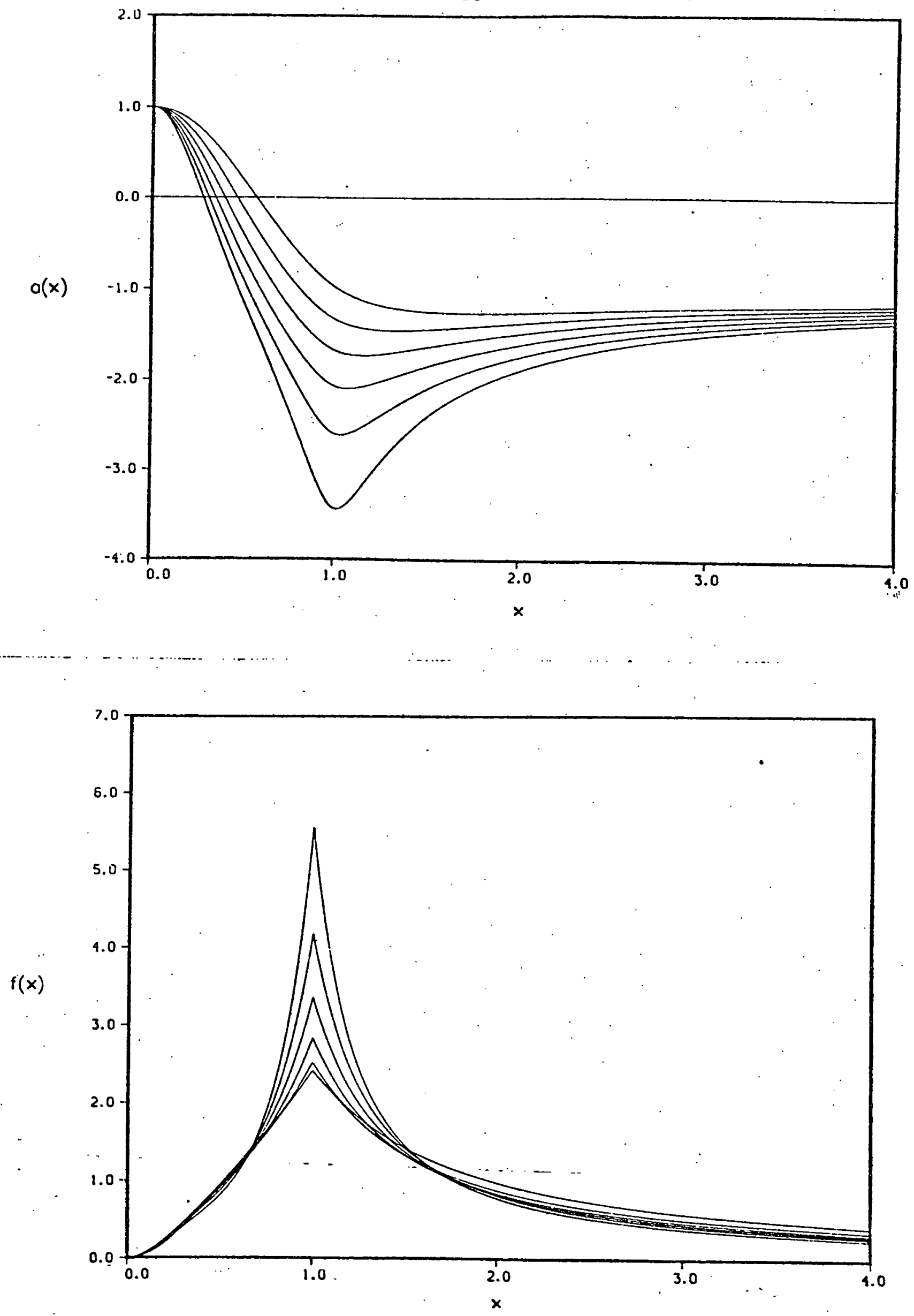

FIG 4 